\title{
Combined Robotic Pyelolithotomy and Laser Lithotripsy for Staghorn Calculi
}

\author{
Achilles Ploumidis, MD, BSc, MSc, PhD, FEBU, Prodromos Philippou, MD, MA, FEBU, \\ Andreas Skolarikos, MD, PhD, FEBU, Assos. Prof., Grigoris Athanasiadis, MD, FEBU, \\ Shailesh Raina, MD, MBBS, MS, D. Urol(lon), Antonios Ploumidis, MD, PhD, FEBU \\ Department of Urology, Athens Medical Center, Athens, Greece (Drs. Ac. Ploumidis, An. Ploumidis). \\ Department of Urology, Apollonion Private Hospital, Nicosia, Cyprus (Dr. Philippou). \\ University Department of Urology, Sismanoglio Hospital, Athens, Greece (Drs. Skolarikos, Athanasiadis). \\ Department of Urology, Jaslok Hospital, Mumbai, India (Dr. Raina).
}

\begin{abstract}
Introduction: Robotic-assisted pyelolithotomy (RAPL) has recently immerged as an alternative approach for the management of complex kidney stones. RAPL combines the benefits of minimally invasive surgery with the high stone clearance rates achieved by open lithotomy.

Case Description: We describe the successful combination of RAPL and Holmium laser lithotripsy for the management of a staghorn calculus in a 57-year-old man who had previously undergone a failed percutaneous nephrolithotomy.

Discussion and Conclusion: The 500- $\mu \mathrm{m}$ Holmium laser fiber was navigated by the console surgeon, who also controlled the laser foot pedal, thus maximizing surgeon control of the procedure. The combination of robotically assisted laparoscopic surgery and Holmium laser lithotripsy is a feasible and safe management option for achieving clearance of complex renal stones. To the best of our knowledge, this appears to be the first reported case, confirming the safety and efficacy of this combined technique.
\end{abstract}

Key Words: Robotic pyelolithotomy, Holmium laser, Staghorn calculus.

Citation Ploumidis A, Philippou P, Skolarikos A, Athanasiadis G, Raina S, Ploumidis A. Combined robotic pyelolithotomy and laser lithotripsy for staghorn calculi. CRSLS e2014.00166. DOI: 10.4293/CRSLS.2014.00166.

Copyright (C) 2014 SLS This is an open-access article distributed under the terms of the Creative Commons Attribution-Noncommercial-ShareAlike 3.0 Unported license, which permits unrestricted noncommercial use, distribution, and reproduction in any medium, provided the original author and source are credited.

Address correspondence to: Achilles Ploumidis, MD, BSc, MSc, FEBU, Department of Urology, Athens, Medical Center, 5-7 Distomou Str., 15125, Athens, Greece. Telephone: (+30) 210-6198100-120, Fax: (+30) 210-6198555, E-mail: aploumidis@yahoo.gr

\section{INTRODUCTION}

The expansion of the endourologic armamentarium has revolutionized the surgical management of urolithiasis, leading to diminished use of open surgery. ${ }^{1}$ According to the European Association of Urology guidelines, however, open lithotomy remains a valid option if a reasonable number of percutaneous approaches are not likely to be successful or if multiple endourologic approaches have unsuccessful results. ${ }^{2}$

Robotic-assisted laparoscopic surgery is increasingly replacing open surgery owing to the wide dissemination of robotic technology, as well as favorable patient outcomes in terms of lower postoperative morbidity, shorter hospital stay and time to convalescence, better cosmetic results, and excellent functional results. In this context, robotic-assisted pyelolithotomy (RAPL) has been described as a safe and effective management option for complex stone burden, especially in the presence of anatomic abnormalities, morbid obesity, or skeletal deformities. ${ }^{3}$ We report our experience in managing a staghorn calculus by combining RAPL and laser stone fragmentation. To our knowledge, this appears to be the first such reported case, confirming the safety and efficacy of this combined technique.

\section{Case Description}

A 57-year-old man with a body mass index of $33 \mathrm{~kg} / \mathrm{m}^{2}$ presented with gross hematuria secondary to a left staghorn calculus. A previous percutaneous nephrolithotomy (PCNL) failed, and the patient was referred to our center for RAPL. 
With the patient under general anesthesia, a nasogastric tube and a urethral catheter on free drainage were inserted. The patient was positioned in the lateral decubitus position at $60^{\circ}$ with his left side facing up. Using Hasson's technique, the camera port was inserted at the lateral edge of the rectus muscle at the level of the umbilicus, and a pneumoperitoneum was established. Two robotic ports were placed under direct laparoscopic vision (one at the midline $3 \mathrm{~cm}$ below the xiphoid process and one at the anterior axillary line $3 \mathrm{~cm}$ below the costal margin). Two additional laparoscopic assisting ports were inserted: a 5-mm port between the camera port and the left robotic port at the midline and a $12-\mathrm{mm}$ port above the midline and caudal to the camera port (for insertion of a retrieval bag).

The line of Toldt was incised and the left colon mobilized and reflected medially, thus exposing the ureteropelvic junction (UPJ). The renal pelvis was mobilized on all sides using sharp and blunt dissection. A longitudinal concave incision with the curve facing the UPJ was made on the posterior surface of the renal pelvis. The stone was identified, but despite our multiple efforts, its volume and geometry precluded its extraction in one piece (Figure 1A).

To overcome this problem, a 500- $\mu$ m Holmium laser fiber (protected within a 6-Fr ureteric catheter) was inserted through an assistant port to facilitate stone fragmentation in manageable pieces (Figure 1B). The surgeon accurately manipulated the laser fiber with the robotic Maryland forceps while having control of the laser foot petal. Intracorporeal lithotripsy was successful, leading to the formation of 2 large stone fragments that were removed using laparoscopic grasping forceps and an endoscopic retrieval bag (Figure 1C and $\mathbf{D}$ ). The renal pelvis was thoroughly inspected for residual stones and copiously irrigated. The absence of residual stone fragments or significant damage to the pelvic mucosa was confirmed. Pyeloplasty was not considered necessary, and watertight closure of the renal pelvis over an antegrade double-J stent was ensured, using 2 half-running 3-0 polyglactin sutures.

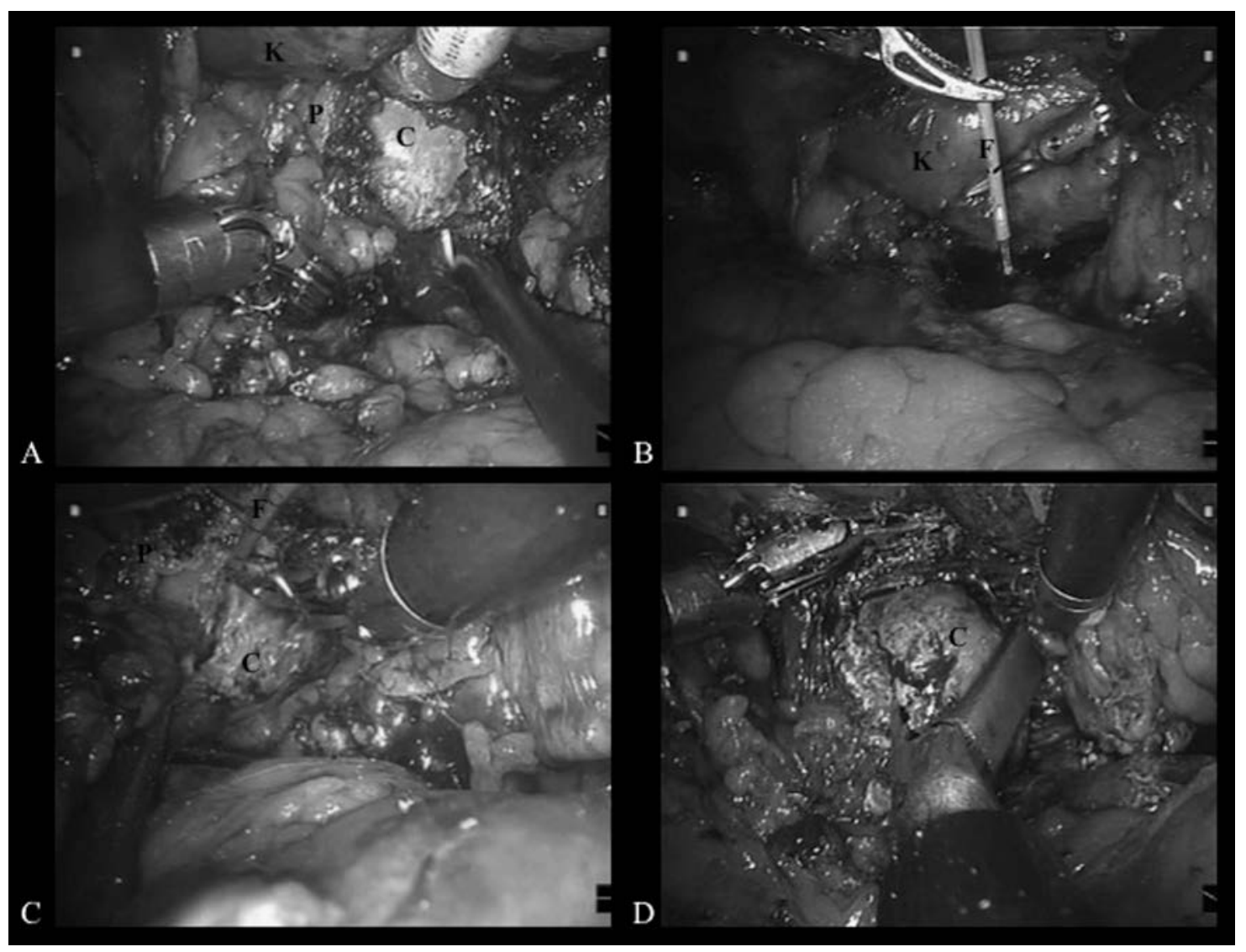

Figure 1. A, Visualization of the calculus after incising the renal pelvis. B, The Holmium laser fiber protected within a ureteric catheter is controlled by the console surgeon. C, Fragmentation of the staghorn calculus. D, Removal of fragments with a laparoscopic grasping forceps. K, kidney; P, renal pelvis; C, calculus; F, Holmium laser fiber. 


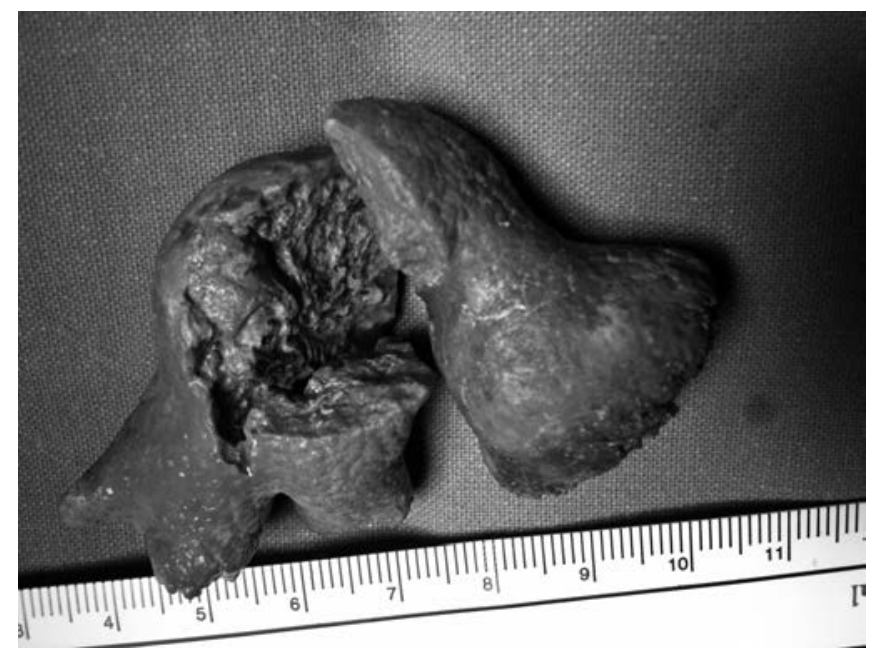

Figure 2. The staghorn calculus extracted in 2 fragments.

At the end of the procedure, a drain was inserted through the caudal robotic port, the robot was undocked, and the ports were removed under direct vision. The endoscopic bag containing the stone fragments (Figure 2) was removed from a small extension of the camera port incision, and the port-site wounds were closed. The operative time was 190 minutes (including robot docking) and intraoperative blood loss was minimal.

The patient's postoperative course was uneventful, and he was discharged on the second postoperative day. A kidneyureter-bladder radiograph performed 2 weeks postoperatively confirmed the absence of residual calculi (Figure 3).

\section{DISCUSSION}

PCNL is the modality of choice for the management of large/staghorn renal calculi, with or without adjuvant extracorporeal shockwave lithotripsy (ESWL). ${ }^{1}$ PCNL, however, has some limitations when used for the treatment of complex stone burden, including the need for multiple access tracts, increased morbidity, longer hospital stay, increased rates of residual stone fragments, and the need for additional PCNL sessions or ancillary procedures. ${ }^{4}$

Open lithotomy overcomes the limitations of PCNL in terms of stone clearance but remains an invasive procedure and is used only as a last resort. ${ }^{2}$ Laparoscopic pyelolithotomy (retroperitoneal or transperitoneal) has been proposed as a minimally invasive alternative to open surgery, with high stone clearance rates (range, 75100\%). ${ }^{5-7}$ According to a retrospective study comparing laparoscopic retroperitoneal pyelolithotomy $(n=16)$ with PCNL $(\mathrm{n}=12),{ }^{8}$ PCNL caused less morbidity and was

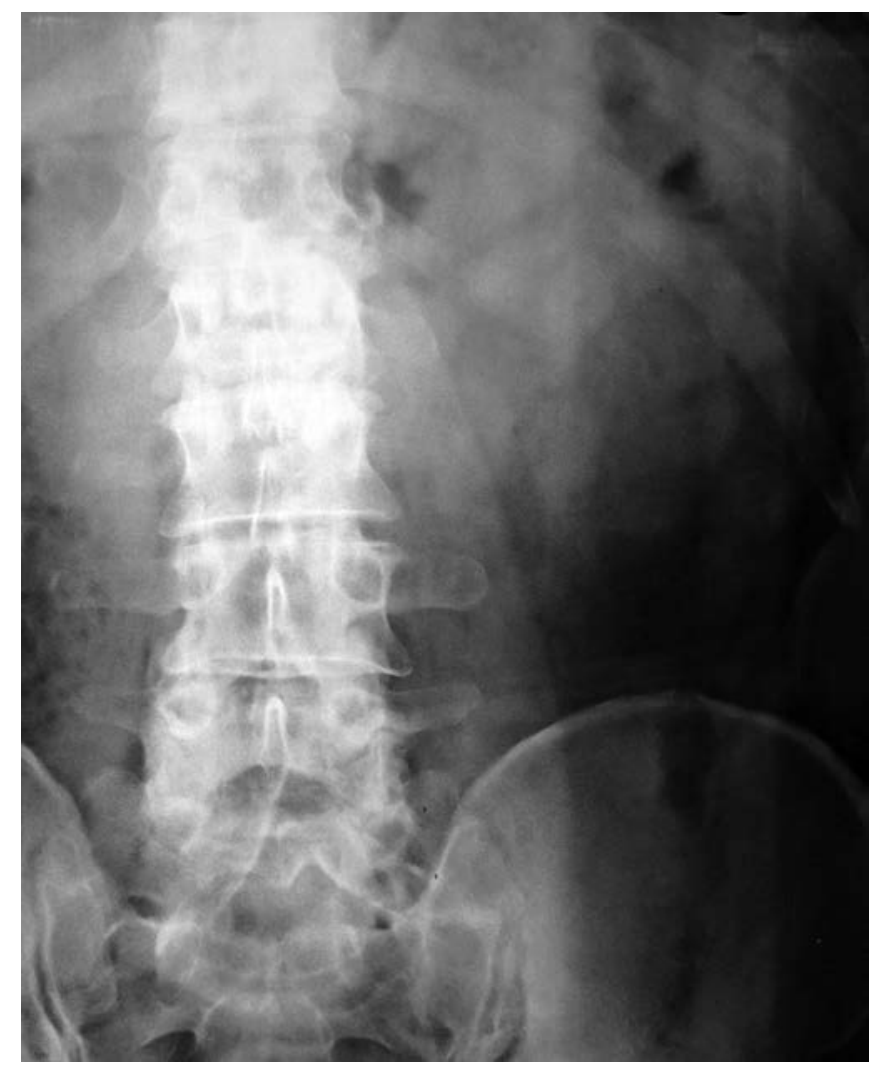

Figure 3. Kidney-ureter-bladder radiograph 2 weeks postoperatively, confirming stone-free status.

easier to perform, whereas laparoscopic pyelolithotomy should be reserved for patients who need adjunctive procedures. A similar retrospective study comparing transperitoneal laparoscopic pyelolithotomy and PCNL also showed that the laparoscopic approach is safe but technically challenging and time consuming. ${ }^{9}$ Laparoscopic anatrophic nephrolithotomy has also been described as an alternative to open surgery for staghorn calculi. ${ }^{10}$ Simforoosh et al reported their experience with 5 complex renal calculi (mean stone size, $53 \mathrm{~mm}$ ), leading to acceptable stone clearance rates (in 2 cases small residual fragments were managed by extracorporeal shockwave lithotripsy). Laparoscopic anatrophic nephrolithotomy, however, may necessitate a relatively longer warm ischemia time. ${ }^{10}$

According to the limited available evidence, laparoscopic surgery for kidney stones is safe and feasible but is also technically challenging and time consuming. ${ }^{8-10}$ To overcome the limitations of conventional laparoscopy, the use of the robotic interface enables minimally invasive stone clearance and precise reconstruction of the pelvicalyceal system. Robotic-assisted extended pyelolithotomy was 
proposed more than 5 years ago as a safe and effective alternative to PCNL for partial staghorn calculi. ${ }^{3,11-13}$ RAPL allows the removal of complex renal stones and the simultaneous reconstruction of UPJ obstruction, ${ }^{14}$ even in difficult cases such as an intrarenal pelvis or an ectopic kidney. ${ }^{15}$ It avoids renal parenchymal transgression and theoretically minimizes renal function loss, and this can be of importance in the context of solitary kidneys or compromised renal function. The necessary instrumentation and surgical steps for RAPL have been described in the past and are summarized in Tables $\mathbf{1}$ and $\mathbf{2} .^{13}$

The traditional approach to open pyelolithotomy was retroperitoneal, but for the laparoscopic or robotic procedure, the transperitoneal approach offers extensive working space, establishes a more convenient angle to inspect the calyces for residual stone, and facilitates closure of the pyelotomy..$^{13}$ However, attention should be paid to watertight closure of the renal pelvis to avoid urine leakage into the peritoneal cavity. Accurate closure of the renal pelvis can be improved using the advantages of the hand-simulating EndoWrist technology (Intuitive Surgical, Sunnyvale, California) of the robotic equipment. ${ }^{3}$

One of the major advantages of pyelolithotomy is removal of the stone without fragmentation, thus minimizing the risk of residual fragments and urosepsis. To remove a large stone through the pyelotomy incision, various manipulations can be used5:

1. Dislodging and manipulating the stone into a position with its smallest diameter lying parallel to the pyelotomy

2. Extending the pyelotomy into the intrarenal pelvis or infundibulum in a controlled fashion

3. Holding the stone with the Maryland robotic forceps or with the assistants grasping the forceps and applying gen-
Table 2.

Summary of Surgical Steps for RAPL

Step 1: Kidney exposure/colon mobilization

Step 2: Exposure of renal pelvis, retraction, and pyelotomy

Step 3: Stone extraction ( \pm Holmium laser assistance)

Step 4: Inspection of the pelvicalyceal system (directly with robotic optic and/or flexible nephroscope)

Step 5: Pyelotomy closure ( \pm pyeloplasty, \pm antegrade double-J stent insertion + drain placement)

tle traction with rotary movements to gently extract the intrarenal part of the stone under vision, ensuring safe disimpaction from the mucosa

If removal of an intact stone is not possible, the need for intracorporeal lithotripsy may arise using one of the available energy sources. Attempting to remove a large stone through a limited pyelotomy should be avoided because it may result in tears or even UPJ avulsion. If a Holmium laser device is not available, pneumatic lithotripsy can be used as an alternative procedure. The pneumatic lithotripter, however, necessitates the use of the rigid nephroscope, which has limited maneuverability and can be associated with a higher risk for mucosal injury and bleeding. The rigid nephroscope is manipulated by the bedside assistant, who must be adequately trained in its use.

In the case described here, we incorporated the use of the Holmium laser to achieve stone fragmentation in 2 large pieces. The $500-\mu \mathrm{m}$ Holmium laser fiber was navigated by the console surgeon, who also controlled the laser foot pedal, thus maximizing surgeon control of the procedure. Additional incisions

Table 1.

List of Necessary Instruments for RAPL

\begin{tabular}{llll}
\hline Right Robotic Arm & Left Robotic Arm & $\begin{array}{l}\text { Patient-side Surgeon } \\
\text { (Laparoscopic Instruments) }\end{array}$ & Additional Equipment \\
\hline Monopolar shears & Maryland bipolar forceps & Suction-irrigation & Flexible nephroscope \\
Needle driver & Needle driver (optional) & Atraumatic grasper & Stone-grasping forceps \\
Maryland bipolar forceps & & Needle driver & Nitinol basket \\
& Laparoscopic scissors & Lithotripsy energy source (ideally \\
& & & Holmium laser) \\
& & & Laparoscopy/C-arm \\
& & & Lapopic U/S probe
\end{tabular}

$\mathrm{U} / \mathrm{S}$, ultrasound. 
toward the calyces were avoided, thus minimizing tissue injury and facilitating the closure of the pyelotomy.

If stone fragments lie in the calyces, flexible nephroscopy to ensure stone clearance is necessary, and the bedside surgeon should be trained in flexible endoscopy and stone-retrieval equipment because simultaneous manipulation of the nephroscope and the robotic arms is usually required. ${ }^{5}$ The nephroscope can be inserted through the most cranial robotic port, which allows direct access to the renal pelvis. The robotic arm is de-docked and the nephroscope is inserted through the rubber seal, thus maintaining the pneumoperitoneum. The flexible nephroscope is navigated into the renal pelvis using the caudal robotic instrument, while the robotic camera provides direct vision. The bedside surgeon navigates the flexible nephroscope while providing continuous suction of the irrigation fluid through the 5-mm assistant port. Ensuring a stone-free outcome can be challenging because the presence of the robotic apparatus makes intraoperative fluoroscopy impractical. Laparoscopic ultrasonography probes can be used as an alternative,$^{15}$ and the pelvicalyceal system is thoroughly flushed after lithotripsy to wash out any gravel or debris.

RAPL, however, has some limitations and should not be considered as a first-line modality for treating urolithiasis. Patients with complete staghorn calculi or those with an intrarenal pelvis are not considered ideal candidates for the robotic approach. 3,11 The absence of haptic feedback hinders the option of a precise nephrotomy directly over a trapped calyceal stone. Retrieval of stone fragments can be challenging, because the entire pelvicalyceal system is difficult to visualize directly through a limited pyelotomy, and flexible nephroscopy may be necessary. ${ }^{3}$ The availability of the robotic apparatus and adjuvant lithotripsy equipment can be restricted because of their high cost. The limited experience with the technique described herein precludes clear recommendations in regard to its role and anticipated results. This represents an important field for future research, and a prospective study that aims to clarify this issue is currently ongoing in our department.

\section{CONCLUSION}

Current endourologic techniques achieve stone clearance for a vast majority of renal stones. In some complex cases, however, the combination of robotically assisted laparoscopy and Holmium laser lithotripsy is safe and effective and may represent a minimally invasive alternative to traditional open lithotomy. As the surgical experience in robotic-assisted reconstruction of the upper tract expands, further studies will explore the role of the robotic interface in the surgical management of difficult renal stone cases.

\section{References:}

1. Hruza M, Schulze M, Teber D, et al. Laparoscopic techniques for removal of renal and ureteral calculi. J Endourol. 2009;23:1713-1718.

2. Turk C, Knoll T, Petrik A, et al. EAU guidelines on urolithiasis. European Association of Urology. 2012. http://www.uroweb.org/gls/ pdf/20_Urolithiasis_LR\%20March\%2013\%202012.pdf. Accessed December 2012.

3. Hemal AK, Nayyar R, Gupta NP, Dorairajan LN. Experience with robotic assisted laparoscopic surgery in upper tract urolithiasis. Can J Urol. 2010;17:5299-5305.

4. Desai M, De Lisa A, Turna B, et al. The clinical research office of the endourological society percutaneous nephrolithotomy global study: staghorn versus nonstaghorn stones. J Endourol. 2011;25:1263-1268.

5. Hemal AK, Goel A, Kumar M, Gupta NP. Evaluation of laparoscopic retroperitoneal surgery in urinary stone disease. $J$ Endourol. 2001;15:701-705.

6. Stein RJ, Turna B, Nguyen MM, et al. Laparoscopic pyeloplasty with concomitant pyelolithotomy: technique and outcomes. J Endourol. 2008;22:1251-1255.

7. Chander J, Dangi AD, Gupta N, Vindal A, Lal P, Ramteke VK. Evaluation of the role of preoperative Double-J ureteral stenting in retroperitoneal laparoscopic pyelolithotomy. Surg Endosc. 2010;24: $1722-1726$.

8. Goel A, Hemal AK. Evaluation of role of retroperitoneoscopic pyelolithotomy and its comparison with percutaneous nephrolithotripsy. Int Urol Nephrol. 2003;35:73-76.

9. Meria P, Milcent S, Desgrandchamps F, et al. Management of pelvic stones larger than $20 \mathrm{~mm}$ : laparoscopic transperitoneal pyelolithotomy or percutaneous nephrolithotomy? Urol Int. 2005;75:322326.

10. Simforoosh N, Aminsharifi A, Tabibi A, et al. Laparoscopic anatrophic nephrolithotomy for managing large staghorn calculi. BJU Int. 2008;101:1293-1296.

11. Badani KK, Hemal AK, Fumo M, et al. Robotic extended pyelolithotomy for treatment of renal calculi: a feasibility study. World J Urol. 2006;24:198-201.

12. Lee RS, Passerotti CC, Cendron M, et al. Early results of robot assisted laparoscopic lithotomy in adolescents. J Urol. 2007;177:23062309.

13. Badalato GM, Hemal AK, Menon M, Badani KK. Current role of robot-assisted pyelolithotomy for the management of large renal calculi: a contemporary analysis. J Endourol. 2009;23:1719-1722.

14. Nayyar R, Gupta NP, Hemal AK. Robotic management of complicated ureteropelvic junction obstruction. World J Urol. 2010;28:599602.

15. Van Cangh PJ, Abi Aad AS, Lorge F, Wese FX, Opsomer R. Laparoscopic nephrolithotomy: the value of intracorporeal sonography and color Doppler. Urology. 1995;45:516-519. 